\title{
MATRIX TRANSFORMATIONS AND GENERATORS OF ANALYTIC SEMIGROUPS
}

\author{
BRUNO DE MALAFOSSE AND AHMED MEDEGHRI
}

Received 3 May 2006; Revised 8 June 2006; Accepted 15 June 2006

We establish a relation between the notion of an operator of an analytic semigroup and matrix transformations mapping from a set of sequences into $\chi$, where $\chi$ is either of the sets $l_{\infty}, c_{0}$, or $c$. We get extensions of some results given by Labbas and de Malafosse concerning applications of the sum of operators in the nondifferential case.

Copyright (C) 2006 B. de Malafosse and A. Medeghri. This is an open access article distributed under the Creative Commons Attribution License, which permits unrestricted use, distribution, and reproduction in any medium, provided the original work is properly cited.

\section{Introduction}

In this paper, we are interested in the study of operators represented by infinite matrices. Note that in [1], Altay and Başar gave some results on the fine spectrum of the difference operator $\Delta$ acting on the sequence spaces $c_{0}$ and $c$. Then they dealt with the fine spectrum of the operator $B(r, s)$ defined by a matrix band over the sequence spaces $c_{0}$ and $c$. In de Malafosse $[3,5]$, there are results on the spectrum of the Cesàro matrix $C_{1}$ and on the matrix $\Delta$ considered as operators from $s_{r}$ to itself. Spectral properties of unbounded operators are used in the theory of the sum of operators. The notion of generators of analytic semigroup was developed in this way. Recall that this theory was studied by many authors such as Da Prato and Grisvard [2, 12], Fuhrman [11], Labbas and Terreni [16, 17]. Some applications can also be found in Labbas and de Malafosse [15] of the sum of operators in the theory of summability in the noncommutative case. Some results were obtained in de Malafosse [4] on the equation

$$
A x+B x-\lambda x=y \quad \text { for } \lambda \geq 0
$$

in a reflexive Banach set of sequences $E$, where $y \in E, A$ and $B$ are two closed linear operators represented by infinite matrices with domains $D(A)$ and $D(B)$ included in $E$.

Here we are interested in some extensions of results given in [15] using similar matrices $A$ and $B$. Recall that the choice of these matrices was motivated by the solvability of 
a class of infinite-tridiagonal systems. Then we study some spectral properties of $A$ and $B$ considered as matrix transformations in the sets $\left(s_{1 / a}, l_{\infty}\right)$ and $\left(s_{1 / \beta}, l_{\infty}\right)$, or $\left(s_{1 / a}^{0}, c_{0}\right)$ and $\left(s_{1 / \beta}^{0}, c_{0}\right)$, or $\left(s_{1 / a}^{(c)}, c\right)$ and $\left(s_{1 / \beta}^{(c)}, c\right)$. Then we show that $(-A)$ and $(-B)$ are generators of analytic semigroups, where $D(A)$ and $D(B)$ are of the form $\chi(A)$ and $\chi(B)$ with $\chi=l_{\infty}, c_{0}$, or $c$. Here the relative boundedness with respect to $A$ or $B$ is not satisfied, so we are not within the framework of the classical perturbation theory given by Kato [14] or Pazy [18].

In this paper, we establish a relation between results in summability and basic notions used in the theory of the sum of operators. For this, we need to recall the following.

\section{Preliminary results}

2.1. Recall of some results in summability. Let $M=\left(a_{n m}\right)_{n, m \geq 1}$ be an infinite matrix and consider the sequence $x=\left(x_{n}\right)_{n \geq 1}$. We will define the product $M x=\left(M_{n}(x)\right)_{n \geq 1}$ with $M_{n}(x)=\sum_{m=1}^{\infty} a_{n m} x_{m}$ whenever the series are convergent for all $n \geq 1$. Let $s$ denote the set of all complex sequences. We write $\varphi, c_{0}, c$ and $l_{\infty}$ for the sets of finite, null, convergent, and bounded sequences, respectively. For any given subsets $X, Y$ of $s$, we will say that the operator represented by the infinite matrix $M=\left(a_{n m}\right)_{n, m \geq 1}$ maps $X$ into $Y$, that is, $M \in(X, Y)$, if the series defined by $M_{n}(x)=\sum_{m=1}^{\infty} a_{n m} x_{m}$ are convergent for all $n \geq 1$ and for all $x \in X$ and $M x \in Y$ for all $x \in X$. For any subset $X$ of $s$, we will write

$$
M X=\{y \in s: y=M x \text { for some } x \in X\} .
$$

If $Y$ is a subset of $s$, we will denote the so-called matrix domain by

$$
Y(M)=\{x \in s: y=M x \in Y\}
$$

Let $X \subset s$ be a Banach space, with norm $\|\cdot\|_{X}$. By $\mathscr{B}(X)$, we will denote the set of all bounded linear operators, mapping $X$ into itself. We will say that $L \in \mathscr{B}(X)$ if and only if $L: X \mapsto X$ is a linear operator and

$$
\|L\|_{\mathscr{B}(X)}^{*}=\sup _{x \neq 0}\left(\|L x\|_{X} /\|x\|_{X}\right)<\infty .
$$

It is well known that $\mathscr{B}(X)$ is a Banach algebra with the norm $\|L\|_{\mathscr{B}(X)}^{*}$. A Banach space $X \subset s$ is a BK space if the projection $P_{n}: x \mapsto x_{n}$ from $X$ into $\mathbb{C}$ is continuous for all $n$. A BK space $X \supset \varphi$ is said to have AK if for every $x \in X, x=\lim _{p \rightarrow \infty} \sum_{k=1}^{p} x_{k} e_{k}$, where $e_{k}=(0, \ldots, 1, \ldots), 1$ being in the $k$ th position. It is well known that if $X$ has $\mathrm{AK}$, then $\mathscr{B}(X)=(X, X)$, see $[9,13,19]$.

Put now $U^{+}=\left\{x=\left(x_{n}\right)_{n \geq 1} \in s: x_{n}>0\right.$ for all $\left.n\right\}$. For $\xi=\left(\xi_{n}\right)_{n \geq 1} \in U^{+}$, we will define the diagonal matrix $D_{\xi}=\left(\xi_{n} \delta_{n m}\right)_{n, m \geq 1}$, (where $\delta_{n m}=0$ for all $n \neq m$ and $\delta_{n m}=1$ otherwise). For $\alpha \in U^{+}$, we will write $s_{\alpha}=D_{\alpha} l_{\infty}$, (cf. [3-10,15]. The set $s_{\alpha}$ is a BK space with the norm $\|x\|_{s_{\alpha}}=\sup _{n \geq 1}\left(\left|x_{n}\right| / \alpha_{n}\right)$. The set of all infinite matrices $M=\left(a_{n m}\right)_{n, m \geq 1}$ with

$$
\|M\|_{S_{\alpha}}=\sup _{n \geq 1}\left(\frac{1}{\alpha_{n}} \sum_{m=1}^{\infty}\left|a_{n m}\right| \alpha_{m}\right)<\infty
$$


is a Banach algebra with identity normed by $\|\cdot\|_{S_{\alpha}}$. Recall that if $M \in\left(s_{\alpha}, s_{\alpha}\right)$, then $\|M x\|_{s_{\alpha}} \leq\|M\|_{S_{\alpha}}\|x\|_{s_{\alpha}}$ for all $x \in s_{\alpha}$. Thus we obtain the following result, (cf. [7]) where we put $B\left(s_{\alpha}\right)=\mathscr{B}\left(s_{\alpha}\right) \cap\left(s_{\alpha}, s_{\alpha}\right)$.

Lemma 2.1. For any given $\alpha \in U^{+}, B\left(s_{\alpha}\right)=S_{\alpha}=\left(s_{\alpha}, s_{\alpha}\right)$.

In the same way, we will define the sets $s_{\alpha}^{0}=D_{\alpha} c_{0}$ and $s_{\alpha}^{(c)}=D_{\alpha} c$, (cf. [7]). The sets $s_{\alpha}^{0}$ and $s_{\alpha}^{(c)}$ are BK spaces with the norm $\|\cdot\|_{s_{\alpha}}$ and $s_{\alpha}^{0}$ has AK. It was shown in $[9,10]$ that for any matrix $M \in\left(s_{\alpha}, s_{\alpha}\right)$, we get

$$
\|M\|_{\mathscr{B}\left(s_{\alpha}\right)}^{*}=\|M\|_{\mathscr{B}\left(s_{\alpha}^{0}\right)}^{*}=\|M\|_{\mathscr{B}\left(s_{\alpha}^{(c)}\right)}^{*}=\|M\|_{S_{\alpha}} .
$$

In all what follows, we will use the next lemma.

Lemma 2.2. Let $\alpha, \beta \in U^{+}$and let $X, Y$ be subsets of $s$. Then

$$
M \in\left(D_{\alpha} X, D_{\beta} Y\right) \quad \text { iff } D_{1 / \beta} M D_{\alpha} \in(X, Y)
$$

2.2. Operator generators of analytic semigroups. We recall here some results given in Da Prato and Grisvard [2] and Labbas and Terreni [16, 17]. Let $E$ be a Banach space. We consider two closed linear operators $A$ and $B$, whose domains are $D(A)$ and $D(B)$ included in $E$. For every $x \in D(A) \cap D(B)$, we then define their sum $S x=A x+B x$.

The spectral properties of $A$ and $B$ are the following:

(H) there are $C_{A}, C_{B}>0$, and $\left.\varepsilon_{A}, \varepsilon_{B} \in\right] 0, \pi[$ such that

$$
\begin{gathered}
\rho(A) \supset \sum_{A}=\left\{z \in \mathbb{C}:|\operatorname{Arg}(z)|<\pi-\varepsilon_{A}\right\}, \\
\left\|(A-z I)^{-1}\right\|_{\mathfrak{E}(E)} \leq \frac{C_{A}}{|z|} \quad \forall z \in \sum_{A}-\{0\}, \\
\rho(B) \supset \sum_{B}=\left\{z \in \mathbb{C}:|\operatorname{Arg}(z)|<\pi-\varepsilon_{B}\right\}, \\
\left\|(B-z I)^{-1}\right\|_{\mathfrak{E}(E)} \leq \frac{C_{B}}{|z|} \quad \forall z \in \sum_{B}-\{0\}, \\
\varepsilon_{A}+\varepsilon_{B}<\pi .
\end{gathered}
$$

It is said that $A$ and $B$ are generators of analytic semigroups not strongly continuous at $t=0$ and we have $\sigma(A) \bigcap \sigma(-B)=\varnothing$ and $\rho(A) \bigcup \rho(-B)=\mathbb{C}$.

The following is well known in the commutative case:

$$
(A-\xi I)^{-1}(B-\eta I)^{-1}-(B-\eta I)^{-1}(A-\xi I)^{-1}=0 \quad \forall \xi \in \rho(A), \eta \in \rho(B),
$$

if $D(A)$ and $D(B)$ are densely defined in $E$, it is well known (cf. [2]) that the bounded operator defined by

$$
L_{\lambda}=-\frac{1}{2 i \pi} \int_{\Gamma}(B+z I)^{-1}(A-\lambda I-z I)^{-1} d z \quad \forall \lambda>0,
$$


4 Matrix and generators of analytic semigroups

where $\Gamma$ is an infinite-sectorial curve lying in $\rho(A-\lambda I) \bigcap \rho(-B)$ coincides with $(\overline{A+B}-$ $\lambda I)^{-1}$.

In the following, we will consider matrix transformations $A$ and $B$ mapping in a set of sequences and we will show that they satisfy hypothesis $(\mathrm{H})$.

\section{Definition of the operators $A$ and $B$}

We will consider two infinite matrices and deal with the case when $A$ and $B$ map into $l_{\infty}$ or $c_{0}$ and with the case when $A$ and $B$ map into $c$. In each case, we will study their spectral properties.

For given sequences $a=\left(a_{n}\right)_{n \geq 1}, b=\left(b_{n}\right)_{n \geq 1}, \beta=\left(\beta_{n}\right)_{n \geq 1}$, and $\gamma=\left(\gamma_{n}\right)_{n \geq 1}$, let $A$ and $B$ be the following infinite matrices:

$$
A=\left[\begin{array}{cccc}
a_{1} & b_{1} & & 0 \\
& \cdot & \cdot & \\
O & & a_{n} & b_{n} \\
& & & \cdot
\end{array}\right], \quad B=\left[\begin{array}{cccc}
\beta_{1} & & & O \\
\cdot & \cdot & & \\
& \gamma_{n} & \beta_{n} & \\
O & & & \cdot
\end{array}\right]
$$

3.1. The case when $A$ and $B$ are operators mapping from $D(A)$ and $D(B)$ into $E$, where $E=l_{\infty}$ or $c_{0}$. The next conditions are consequences of results given in [15]. When $E$ is either of the sets $l_{\infty}$ or $c_{0}$, we assume that $A$ satisfies the following properties:

$$
\begin{gathered}
a \in U^{+}, \quad a_{n} \text { is strictly increasing, } \lim _{n \rightarrow \infty} a_{n}=\infty, \\
\text { there is } M_{A}>0 \quad \text { such that }\left|b_{n}\right| \leq M_{A} \forall n .
\end{gathered}
$$

Similarly, we assume that $B$ satisfies the next conditions:

$$
\begin{gathered}
\beta \in U^{+}, \quad \lim _{k \rightarrow \infty} \beta_{2 k}=L \neq 0, \\
\lim _{k \rightarrow \infty} \frac{\beta_{2 k+1}}{a_{2 k+1}}=\infty,
\end{gathered}
$$

$(\alpha)$ there is $M_{B}>0 \quad$ such that $\left|\gamma_{2 k}\right| \leq M_{B} \forall n, \quad(\beta) \gamma_{2 k+1}=o(1) \quad(n \longrightarrow \infty)$.

3.2. The case when $A$ and $B$ are operators mapping $D(A)$ and $D(B)$ into $c$. Here we need to recall the characterization of $(c, c)$.

Lemma 3.1. $A \in(c, c)$ if and only if

(i) $A \in S_{1}$,

(ii) $\lim _{n \rightarrow \infty} \sum_{m=1}^{\infty} a_{n m}=$ l for some $l \in \mathbb{C}$,

(iii) $\lim _{n \rightarrow \infty} a_{n m}=l_{m}$ for some $l_{m} \in \mathbb{C}, m=1,2, \ldots$

We will see that $D(A)=c(A)=s_{1 / a}^{(c)}$ and $D(B)=c(B)=s_{1 / \beta}^{(c)}$. Here we will show that neither of the sets $D(A)=s_{1 / a}^{(c)}$ and $D(B)=s_{1 / \beta}^{(c)}$ is embedded in the other. 
Proposition 3.2. Let $a, \beta \in U^{+}$. Then

$$
s_{1 / a}^{(c)} \nsubseteq s_{1 / \beta}^{(c)}, \quad s_{1 / \beta}^{(c)} \nsubseteq s_{1 / a}^{(c)}
$$

if and only if $\beta / a, a / \beta \notin c$.

Proof. The inclusion $s_{1 / a}^{(c)} \subset s_{1 / \beta}^{(c)}$ means that $I \in\left(s_{1 / a}^{(c)}, s_{1 / \beta}^{(c)}\right)$ and by Lemma 2.2, we have $D_{\beta / a} \in(c, c)$. From Lemma 3.1, we conclude that $s_{1 / a}^{(c)} \subset s_{1 / \beta}^{(c)}$ if and only if $\beta / a \in c$. So $s_{1 / a}^{(c)} \nsubseteq s_{1 / \beta}^{(c)}$ is equivalent to $\beta / a \notin c$. Similarly, we have $s_{1 / \beta}^{(c)} \nsubseteq s_{1 / a}^{(c)}$ if and only if $a / \beta \notin c$. This completes the proof.

We assume that $A$ and $B$ satisfy the following hypotheses. The matrix $A$ is defined in (3.1) with

$$
\begin{gathered}
a \in U^{+}, \quad a_{n} \text { is strictly increasing, } \lim _{n \rightarrow \infty} a_{n}=\infty, \\
b \in c .
\end{gathered}
$$

For $B$ given in (3.1), we do the following hypotheses:

$$
\begin{gathered}
\beta \in U^{+}, \quad \lim _{n \rightarrow \infty} \beta_{n}=\infty, \\
\lim _{k \rightarrow \infty} \frac{\beta_{2 k+1}}{a_{2 k+1}}=\infty, \quad \lim _{k \rightarrow \infty} \frac{\beta_{2 k}}{a_{2 k}}=l \neq 0, \\
\gamma \in c .
\end{gathered}
$$

This lead to the next remark.

Remark 3.3. The choice of $\beta$ in (3.6b) is justified by Proposition 3.2 and so neither of the sets $D(A)=s_{1 / a}^{(c)}$ and $D(B)=s_{1 / \beta}^{(c)}$ is embedded in the other one. We will see in Proposition 5.7 and Theorem 5.8 that we need to have (3.5a) and (3.6a). Then we will see that $A$ and $B$ are closed operators when $b, \gamma \in c$. Finally, notice that $D(B) \subset c$ means $1 / \beta \in c$ which is trivially satisfied in (3.6a) and it is the same for $A$.

\section{First properties of the operators $A$ and $B$}

4.1. The case when the operators $A$ and $B$ are considered as matrix maps from $D(A)$ and $D(B)$ into $E$, where $E$ is equal to $l_{\infty}$, or $c_{0}$. In this section, we will assume $A$ and $B$ satisfy (3.2) and (3.3). For the convenience of the reader, recall the following well-known results.

Lemma 4.1. (i) $A \in\left(l_{\infty}, l_{\infty}\right)$ if and only if $A \in S_{1}$.

(ii) $A \in\left(c_{0}, c_{0}\right)$ if and only if $A \in S_{1}$ and $\lim _{n \rightarrow \infty} a_{n m}=0$ for each $m=1,2, \ldots$.

Proposition 4.2. (i) $A \in\left(s_{1 / a}, l_{\infty}\right)$ and $A \in\left(s_{1 / a}^{0}, c_{0}\right)$.

(ii) $B \in\left(s_{1 / \beta}, l_{\infty}\right)$ and $B \in\left(s_{1 / \beta}^{0}, c_{0}\right)$. 
Proof. (i) We have $\left[A D_{1 / a}\right]_{n n}=1$ and $\left[A D_{1 / a}\right]_{n, n+1}=b_{n} / a_{n+1}$ for all $n$, and $\left[A D_{1 / a}\right]_{n m}=0$ otherwise. Then

$$
\left\|A D_{1 / a}\right\|_{S_{1}}=\sup _{n}\left(1+\frac{\left|b_{n}\right|}{a_{n+1}}\right)=O(1) \quad(n \longrightarrow \infty) .
$$

So by Lemma 4.1, we have $A D_{1 / a} \in\left(l_{\infty}, l_{\infty}\right)$ and by Lemma $2.2, A \in\left(s_{1 / a}, l_{\infty}\right)$. The proof is similar for $A \in\left(s_{1 / a}^{0}, c_{0}\right)$ note that in this case, $\left[A D_{1 / a}\right]_{n m} \rightarrow 0(n \rightarrow \infty)$ for each $m \geq 1$.

(ii) Now $\left[B D_{1 / \beta}\right]_{n n}=1$ and $\left[B D_{1 / \beta}\right]_{n, n-1}=\gamma_{n} / \beta_{n-1}$ for all $n$, and $\left[B D_{1 / \beta}\right]_{n m}=0$ otherwise. By (3.3a), (3.3c) $(\beta)$, we have

$$
\frac{\gamma_{2 n+1}}{\beta_{2 n}}=o(1) \quad(n \rightarrow \infty)
$$

and by $(3.2 \mathrm{a}),(3.3 \mathrm{~b})$ and $(3.3 \mathrm{c})(\alpha)$, we get

$$
\frac{\gamma_{2 n}}{\beta_{2 n-1}}=\frac{\gamma_{2 n}}{a_{2 n-1}} \frac{a_{2 n-1}}{\beta_{2 n-1}}=o(1) \quad(n \rightarrow \infty) .
$$

Then

$$
\left\|B D_{1 / \beta}\right\|_{S_{1}}=\sup _{n}\left(1+\frac{\left|\gamma_{n}\right|}{\beta_{n-1}}\right)=O(1) \quad(n \longrightarrow \infty) .
$$

So $B D_{1 / \beta} \in\left(l_{\infty}, l_{\infty}\right)$ and $B \in\left(s_{1 / \beta}, l_{\infty}\right)$. Finally, we obtain $B \in\left(s_{1 / \beta}^{0}, c_{0}\right)$ reasoning as above.

We deduce that if $E=l^{\infty}=s_{1}$, the matrix $A$ is defined on $D(A)=s_{1 / a}$ and $B$ is defined on $D(B)=s_{1 / \beta}$. It can be shown that $l_{\infty}(A)=s_{1 / a}$ and $l_{\infty}(B)=s_{1 / \beta}$. When $E=c_{0}$, we will see in Theorem 5.6(i), (ii) that $D(A)=c_{0}(A)=s_{1 / a}^{0}$ and $D(B)=c_{0}(B)=s_{1 / \beta}^{0}$. We deduce from (3.3a), (3.3b) that in each case, neither of the sets $D(A)$ and $D(B)$ is embedded in the other.

4.2. The case when the operators $A$ and $B$ are considered as matrix maps from $D(A)$ and $D(B)$ into $c$. We assume that $A$ and $B$ satisfy (3.5) and (3.6). From the preceding, we immediately get the following.

Proposition 4.3. $A \in\left(s_{1 / a}^{(c)}, c\right)$ and $B \in\left(s_{1 / \beta}^{(c)}, c\right)$.

Proof. It is enough to notice that by (3.5) we have $\left(1+b_{n} / a_{n+1}\right)_{n \geq 1} \in c$. Then from Lemma 3.1, we conclude that $A \in\left(s_{1 / a}^{(c)}, c\right)$. We also have by (3.6),

$$
\frac{\gamma_{n}}{\beta_{n-1}} \longrightarrow 0 \quad(n \rightarrow \infty)
$$

so $B D_{1 / \beta} \in(c, c)$ and $B \in\left(s_{1 / \beta}^{(c)}, c\right)$.

We will see in Theorem 5.8(i), (ii) that $D(A)=c(A)=s_{1 / a}^{(c)}$ and $D(B)=c(B)=s_{1 / \beta}^{(c)}$. 


\section{The matrices $A$ and $B$ as operator generators of an analytic semigroup}

In this section, we will show that $A$ and $B$ are generators of analytic semigroup in each case $E=l_{\infty}, E=c_{0}$, or $E=c$.

5.1. The case when $A$ and $B$ are considered as matrix maps from $D(A)$ and $D(B)$ into $E$, where $E=l_{\infty}$ or $c_{0}$. In this section, $A$ and $B$ satisfy (3.2) and (3.3). The next result was shown in [15] in the case when $A \in\left(s_{1 / a}, l_{\infty}\right)$ and $B \in\left(s_{1 / \beta}, l_{\infty}\right)$ with $a_{n}=a^{n}, a>1$, and $\beta$ was defined by $\beta_{2 n}=1$ and $\beta_{2 n+1}=(2 n+1)$ ! for all $n$, so we omit the proof.

Proposition 5.1. In the space $l_{\infty}$, the two linear operators $A$ and $B$ are closed and satisfy the following:

(i) $D(A)=s_{1 / a}$,

(ii) $D(B)=s_{1 / \beta}$,

(iii) $\overline{D(A)} \neq l_{\infty}, \overline{D(B)} \neq l_{\infty}$,

(iv) there are $\varepsilon_{A}, \varepsilon_{B}>0$ (with $\varepsilon_{A}+\varepsilon_{B}<\pi$ ) such that

$$
\begin{gathered}
\left\|(A-\lambda I)^{-1}\right\|_{\mathscr{B}\left(l_{\infty}\right)}^{*} \leq \frac{M}{|\lambda|} \quad \forall \lambda \neq 0, \quad|\operatorname{Arg}(\lambda)| \geq \varepsilon_{A}, \\
\left\|(B+\mu I)^{-1}\right\|_{\mathscr{B}\left(l_{\infty}\right)}^{*} \leq \frac{M}{|\mu|} \quad \forall \mu \neq 0, \quad|\operatorname{Arg}(\mu)| \leq \pi-\varepsilon_{B} .
\end{gathered}
$$

This result shows that $-A$ and $-B$ satisfy hypothesis $(\mathrm{H})$ and $\sigma(-A) \cap \sigma(B)=\varnothing$. So $-A$ and $-B$ are generators of the analytic semigroups $e^{(-A t)}$ and $e^{(-B t)}$ not strongly continuous at $t=0$. We have similar results when $A$ and $B$ are matrix maps into $c_{0}$. We require some elementary lemmas whose proofs are left to the reader.

Lemma 5.2. Let $\varepsilon \in] 0, \pi / 2\left[\right.$ and let $x_{0}>0$ be a real. Then

$$
\left|x_{0}-\lambda\right| \geq x_{0} \sin \varepsilon \quad \forall \lambda \in \mathbb{C} \text { with }|\operatorname{Arg}(\lambda)| \geq \varepsilon
$$

Lemma 5.3. Let $x_{0}>0$ be a real. Then

$$
\begin{gathered}
\left|x_{0}-\lambda\right| \geq|\lambda| \sin \theta \quad \forall \lambda=|\lambda| e^{i \theta} \notin \mathbb{R}^{-}, \\
\left|x_{0}-\lambda\right| \geq|\lambda| \quad \forall \lambda \in \mathbb{R}^{-} .
\end{gathered}
$$

We can state the following result where we will use the fact that for any $\alpha \in U^{+}$, since $s_{\alpha}^{0}$ is a BK space with $\mathrm{AK}$, we have $\mathscr{B}\left(s_{\alpha}^{0}\right)=\left(s_{\alpha}^{0}, s_{\alpha}^{0}\right)$. As we have seen in (2.5), for any matrix $C \in\left(s_{\alpha}^{0}, s_{\alpha}^{0}\right)$, we have $\|C\|_{\mathscr{B}\left(s_{\alpha}^{0}\right)}^{*}=\|C\|_{\left(s_{\alpha}^{0}, s_{\alpha}^{0}\right)}^{*}=\|C\|_{S_{\alpha}}$.

Proposition 5.4. (i) Let $\left.\varepsilon_{A} \in\right] 0, \pi / 2\left[\right.$. For every $\lambda \in \mathbb{C}$ with $|\operatorname{Arg}(\lambda)| \geq \varepsilon_{A}$, the infinite matrix $A-\lambda I$ considered as an operator in $s_{1 / a}^{0}$ is invertible and

$$
(A-\lambda I)^{-1} \in\left(c_{0}, s_{1 / a}^{0}\right)
$$


8 Matrix and generators of analytic semigroups

(ii) Let $\left.\varepsilon_{B} \in\right] 0, \pi / 2\left[\right.$. For every $\mu \in \mathbb{C}$ with $|\operatorname{Arg}(\mu)| \leq \pi-\varepsilon_{B}$, the infinite matrix $B+\mu I$ considered as an operator in $s_{1 / \beta}^{0}$ is invertible and

$$
(B+\mu I)^{-1} \in\left(c_{0}, s_{1 / \beta}^{0}\right) .
$$

Proof. (i) Fix $\left.\varepsilon_{A} \in\right] 0, \pi / 2[$ and consider the infinite-sectorial set

$$
\prod_{\varepsilon_{A}}=\left\{\lambda \in \mathbb{C}:|\operatorname{Arg}(\lambda)|<\varepsilon_{A}\right\} .
$$

For any $\lambda \notin \prod_{\varepsilon_{A}}$, put

$$
\chi_{n}=\frac{b_{n}}{a_{n}-\lambda}
$$

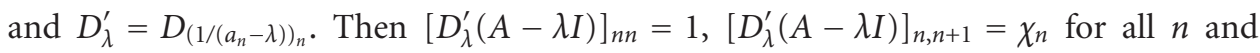
$\left[D_{\lambda}^{\prime}(A-\lambda I)\right]_{n m}=0$ otherwise. By Lemma 5.2, we have

$$
\left|\chi_{n}\right| \leq \frac{M_{A}}{a_{n} \sin \varepsilon_{A}} \quad \forall n \text { and all } \lambda \notin \prod_{\varepsilon_{A}} .
$$

Since $a_{n}$ tends to infinity as $n$ tends to infinity, there is $n_{0}$ such that

$$
\left|\chi_{n}\right| \leq \frac{1}{2} \quad \forall n \geq n_{0} \text { and all } \lambda \notin \prod_{\varepsilon_{A}} .
$$

Consider now the infinite matrix

$$
\widetilde{T}_{\lambda}=\left(\begin{array}{ccccccc} 
& & \cdot & & & \\
& T_{\lambda}^{-1} & \cdot & & & 0 & \\
\cdot & \cdot & & & & & \\
& & & 1 & & \\
\\
0 & & & 1 & & \\
& & & & & \cdot & \\
& & & & & & \\
& & & & &
\end{array}\right)
$$

where $T_{\lambda}$ is the matrix of order $n_{0}$ defined by $\left[T_{\lambda}\right]_{n n}=1$ for $1 \leq n \leq n_{0} ;\left[T_{\lambda}\right]_{n, n+1}=\chi_{n}$ for $1 \leq n \leq n_{0}-1$, and $\left[T_{\lambda}\right]_{n m}=0$ otherwise. Elementary calculations give

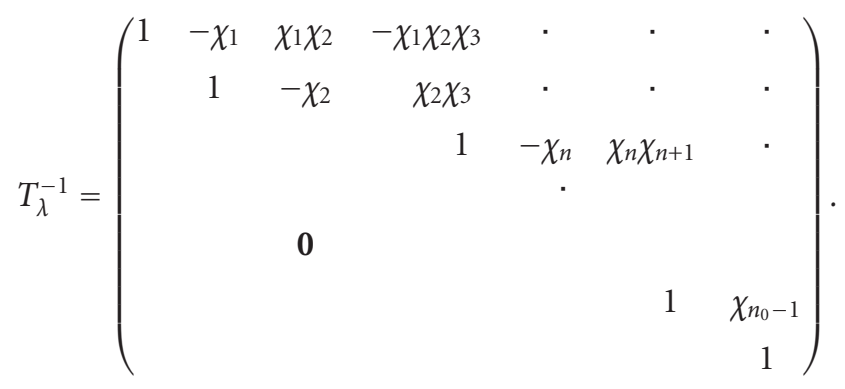


Putting $\mathscr{A}_{\lambda}=D_{\lambda}^{\prime}(A-\lambda I) \widetilde{T}_{\lambda}$ we easily get $\left[\mathscr{A}_{\lambda}\right]_{n n}=1$ for all $n,\left[\mathscr{A}_{\lambda}\right]_{n, n+1}=\chi_{n}$ for $n \geq n_{0}$, and $\left[\mathscr{A}_{\lambda}\right]_{n m}=0$ otherwise. Then by Lemma 2.2 and since the sequence $a$ is increasing, we get

$$
\left\|I-\mathscr{A}_{\lambda}\right\|_{\left(s_{1 / a}^{0}, s_{1 / a}^{0}\right)}^{*}=\left\|I-\mathscr{A}_{\lambda}\right\|_{S_{1 / a}}=\sup _{n \geq n_{0}}\left(\left|\chi_{n}\right| \frac{a_{n}}{a_{n+1}}\right) \leq \frac{1}{2} \quad \forall \lambda \notin \prod_{\varepsilon_{A}} .
$$

Since $\mathscr{A}_{\lambda}=\mathscr{A}_{\lambda}-I+I \in\left(s_{1 / a}^{0}, s_{1 / a}^{0}\right)$ and (5.12) holds, $\mathscr{A}_{\lambda}$ is invertible in the Banach algebra of all bounded operators $\mathscr{B}_{(}\left(s_{1 / a}^{0}\right)=\left(s_{1 / a}^{0}, s_{1 / a}^{0}\right)$ mapping $s_{1 / a}^{0}$ to itself and $\mathscr{A}_{\lambda}^{-1} \in$ $\left(s_{1 / a}^{0}, s_{1 / a}^{0}\right)$. Then for any given $y \in c_{0}$, we successively get $y^{\prime}=D_{\lambda}^{\prime} y=\left(y_{n} /\left(a_{n}-\lambda\right)\right)_{n \geq 1} \in$ $s_{1 / a}^{0}, \mathscr{A}_{\lambda}^{-1} y^{\prime} \in s_{1 / a}^{0}, \widetilde{T}_{\lambda}\left(\mathscr{A}_{\lambda}^{-1} y^{\prime}\right) \in s_{1 / a}^{0}$, and $(A-\lambda I)^{-1}=\widetilde{T}_{\lambda} \mathscr{A}_{\lambda}^{-1} D_{\lambda}^{\prime} \in\left(c_{0}, s_{1 / a}^{0}\right)$. So we have shown (i).

(ii) For $\left.\varepsilon_{B} \in\right] 0, \pi / 2\left[\right.$, let $\Sigma_{B}=\left\{\mu \in \mathbb{C}:|\operatorname{Arg}(\mu)| \leq \pi-\varepsilon_{B}\right\}$ and put

$$
\chi_{n}^{\prime}=\frac{\gamma_{n}}{\beta_{n}+\mu} .
$$

To deal with the inverse of $B+\mu I$, we need to study the sequences $\left|\gamma_{2 k+1}\right| / \beta_{2 k}$ and $\left|\chi_{2 k}^{\prime}\right| \beta_{2 k} \mid$ $\beta_{2 k-1}$. By (3.3a), (3.3b), we have

$$
\frac{\gamma_{2 k+1}}{\beta_{2 k}} \longrightarrow 0 \quad(k \rightarrow \infty) .
$$

On the other hand, for every $\mu \in \Sigma_{B}$, we get

$$
\begin{gathered}
\left|\chi_{2 k}^{\prime}\right| \frac{\beta_{2 k}}{\beta_{2 k-1}} \leq \frac{M_{B}}{\beta_{2 k} \sin \varepsilon_{B}} \frac{\beta_{2 k}}{\beta_{2 k-1}}=\frac{M_{B}}{\sin \varepsilon_{B}} \frac{1}{\beta_{2 k-1}}, \\
\frac{1}{\beta_{2 k-1}}=\frac{a_{2 k-1}}{\beta_{2 k-1}} \frac{1}{a_{2 k-1}}=o(1) \quad(k \longrightarrow \infty) .
\end{gathered}
$$

From (5.14) and (5.16), we deduce that there is $n_{1}$ such that

$$
\begin{aligned}
& \left|\gamma_{2 k+1}\right| \frac{1}{\beta_{2 k}} \leq \frac{1}{2} \sin \varepsilon_{B} \quad \text { for } 2 k+1 \geq n_{1}, \\
& \left|\chi_{2 k}^{\prime}\right| \frac{\beta_{2 k}}{\beta_{2 k-1}} \leq \frac{1}{2} \quad \text { for } 2 k \geq n_{1} \forall \mu \in \Sigma_{B} .
\end{aligned}
$$

As in (i), define the matrices $D_{\mu}^{\prime}=D_{\left(1 /\left(\beta_{n}+\mu\right)_{n}\right)}$ and

$$
\widetilde{R}_{\mu}=\left(\begin{array}{ccccccc} 
& & \cdot & & & & \\
& R_{\mu}^{-1} & \cdot & & & 0 & \\
\cdot & \cdot & \cdot & & & & \\
& & 1 & & & \\
0 & & & 1 & & \\
& & & & & \cdot & \\
& & & & & & \cdot
\end{array}\right) \text {, }
$$


where $R_{\mu}$ is the matrix of order $n_{1}-1$ defined by $\left[R_{\mu}\right]_{n n}=1$ for all $n$, by $\left[R_{\mu}\right]_{n, n-1}=\chi_{n}^{\prime}$ for $2 \leq n \leq n_{1}-1$, and by $\left[R_{\mu}\right]_{n m}=0$ otherwise. Then elementary calculations show that the matrix $\mathscr{B}_{\mu}=\widetilde{R}_{\mu} D_{\mu}^{\prime}(B+\mu I)$ is defined by $\left[\mathscr{B}_{\mu}\right]_{n n}=1$ for all $n$, by $\left[\mathscr{B}_{\mu}\right]_{n, n-1}=\chi_{n}^{\prime}$ for $n \geq n_{1}$, and by $\left[\mathscr{S}_{\mu}\right]_{n m}=0$ otherwise. Then for any $\mu \in \Sigma_{B}$,

$$
\left\|I-\mathscr{B}_{\mu}\right\|_{\left(s_{1 / \beta}^{0}, s_{1 / \beta}^{0}\right)}^{*}=\sup _{n \geq n_{1}}\left(\left|\chi_{n}^{\prime}\right| \frac{\beta_{n}}{\beta_{n-1}}\right)=\max \left(\tau_{1}, \tau_{2}\right)
$$

where

$$
\tau_{1}=\sup _{k \geq n_{1} / 2}\left|\chi_{2 k}^{\prime}\right| \frac{\beta_{2 k}}{\beta_{2 k-1}}, \quad \tau_{2}=\sup _{k \geq\left(n_{1}-1\right) / 2}\left|\chi_{2 k+1}^{\prime}\right| \frac{\beta_{2 k+1}}{\beta_{2 k}} .
$$

By Lemma 5.2, we get $\left|\beta_{2 k}+\mu\right| \geq \beta_{2 k} \sin \varepsilon_{B}$ for all $\mu \in \Sigma_{B}$ and $\tau_{1} \leq 1 / 2$. Then

$$
\tau_{2} \leq \frac{1}{2} \sin \varepsilon_{B} \frac{1}{\beta_{2 k+1} \sin \varepsilon_{B}} \beta_{2 k+1}=\frac{1}{2} \quad \text { for } 2 k \geq n_{1} .
$$

This implies $\left\|I-\mathscr{B}_{\mu}\right\|_{\left(s_{1 / \beta}^{0}, s_{1 / \beta}^{0}\right)}^{*} \leq 1 / 2$. Reasoning as in (i) with $(B+\mu I)^{-1}=\mathscr{B}_{\mu}^{-1} \widetilde{R}_{\mu} D_{\mu}^{\prime}$, we conclude that $B+\mu I$ considered as an operator from $s_{1 / \beta}^{0}$ into $c_{0}$ is invertible and $(B+$ $\mu I)^{-1} \in\left(c_{0}, s_{1 / \beta}^{0}\right)$ for all $\mu \in \Sigma_{B}$. This concludes the proof.

Remark 5.5. As a direct consequence of the preceding, it is trivial that

$$
\begin{gathered}
\mathcal{c}_{0}(A-\lambda I)=s_{1 / a}^{0} \quad \forall \lambda \in \mathbb{C}, \quad|\operatorname{Arg}(\lambda)| \geq \varepsilon_{A}, \\
\mathcal{c}_{0}(B+\mu I)=s_{1 / \beta}^{0} \quad \forall \mu \in \mathbb{C}, \quad|\operatorname{Arg}(\mu)| \leq \pi-\varepsilon_{B} .
\end{gathered}
$$

We immediately obtain the next result.

Theorem 5.6. In the space $c_{0}$, the two linear operators $A$ and $B$ are closed and satisfy the following:

(i) $D(A)=c_{0}(A)=s_{1 / a}^{0}$,

(ii) $D(B)=c_{0}(B)=s_{1 / \beta}^{0}$,

(iii) $\overline{D(A)} \neq c_{0}, \overline{D(B)} \neq c_{0}$,

(iv) there are $\varepsilon_{A}, \varepsilon_{B}>0$ (with $\varepsilon_{A}+\varepsilon_{B}<\pi$ ) such that

$$
\begin{gathered}
\left\|(A-\lambda I)^{-1}\right\|_{\mathscr{B}\left(c_{0}\right)}^{*} \leq \frac{M}{|\lambda|} \quad \forall \lambda \neq 0, \quad|\operatorname{Arg}(\lambda)| \geq \varepsilon_{A}, \\
\left\|(B+\mu I)^{-1}\right\|_{\mathscr{B}\left(c_{0}\right)}^{*} \leq \frac{M}{|\mu|} \quad \forall \mu \neq 0, \quad|\operatorname{Arg}(\mu)| \leq \pi-\varepsilon_{B} .
\end{gathered}
$$

Proof. Show that $A$ is a closed operator. For this, consider a sequence $x_{p}^{\prime}=\left(x_{n p}\right)_{n \geq 1}$ tending to $x=\left(x_{n}\right)_{n \geq 1}$ in $c_{0}$, as $p$ tends to infinity, where $x_{p}^{\prime} \in s_{1 / a}^{0}$ for all $p$. Then $A x_{p}^{\prime} \rightarrow y(p \rightarrow$ $\infty)$ in $c_{0}$, that is for any $n$, we have $A_{n}\left(x_{p}^{\prime}\right) \rightarrow A_{n}(x)=y_{n}(p \rightarrow \infty)$. It remains to show that $x \in s_{1 / a}^{0}$. For this, note that since $b \in l_{\infty}$ and $x \in c_{0}$, we conclude that $a_{n} x_{n}=y_{n}-b_{n} x_{n+1}$ tends to a zero as $n$ tends to infinity. The proof for $B$ is similar. 
(i) $\Rightarrow$ (ii). First by Proposition 4.2, we have $A \in\left(s_{1 / a}^{0}, c_{0}\right)$. It remains to show that $c_{0}(A)=s_{1 / a}^{0}$. Let $x \in s_{1 / a}^{0}$. Since $\left(b_{n} / a_{n+1}\right)_{n \geq 1} \in c_{0}$, we deduce that

$$
A_{n}(x)=a_{n} x_{n}+\frac{b_{n}}{a_{n+1}} a_{n+1} x_{n+1}=o(1) \quad(n \longrightarrow \infty)
$$

and we have shown that $s_{1 / a}^{0} \subset c_{0}(A)$.

Now let $x \in c_{0}(A)$. Then $y=A x \in c_{0}$. In the proof of Proposition 5.4, we can take $\lambda=0$. Indeed, there is $n_{0}$ such that $\chi_{n}=\left|b_{n}\right| / a_{n} \leq 1 / 2$ for all $n \geq n_{0}$. Then $y \in c_{0}$ implies $D_{1 / a} y=\left(y_{n} / a_{n}\right)_{n \geq 1} \in s_{1 / a}^{0}, \mathscr{A}^{-1} D_{1 / a} y \in s_{1 / a}^{0}$, and $x=A^{-1} y=\widetilde{T}_{0} \mathscr{A}_{0}^{-1} D_{1 / a} y \in s_{1 / a}^{0}$. This shows that $c_{0}(A) \subset s_{1 / a}^{0}$ and since $s_{1 / a}^{0} \subset c_{0}(A)$, we conclude that $c_{0}(A)=s_{1 / a}^{0}$. The proof is similar for $B$.

(iii) Let $x=\left(1 / a_{n}\right)_{n \geq 1} \in c_{0}$ and assume $x_{p}^{\prime}=\left(x_{n p}\right)_{n \geq 1}$ tend to $x$ in $s_{1 / a}^{0}$, that is,

$$
\left\|x_{p}^{\prime}-x\right\|_{s_{1 / a}}=\sup _{n}\left(a_{n}\left|x_{n p}-\frac{1}{a_{n}}\right|\right) \longrightarrow 0 \quad(p \longrightarrow \infty) .
$$

Since $a_{n}$ tends to infinity, we should have $x_{n p} \rightarrow 1 / a_{n}$ and $a_{n} x_{n p} \rightarrow 1(p \rightarrow \infty)$ for all $n$. This contradicts the fact that $x_{p}^{\prime} \in s_{1 / a}^{0}$ for all $p$. The reasoning is the same for $B$. So (iii) holds.

(iv) By Proposition 5.4(i), we have seen that for any $\lambda \notin \prod_{\mathcal{E}_{A}}(A-\lambda I)^{-1} \in\left(c_{0}, s_{1 / a}^{0}\right)$, $\widetilde{T}_{\lambda} \in S_{1}$ and using the notation of the proof of Proposition 5.4, we get

$$
\left\|(A-\lambda I)^{-1} y\right\|_{l_{\infty}}=\left\|\tilde{T}_{\lambda} \mathscr{A}_{\lambda}^{-1} y^{\prime}\right\|_{l_{\infty}} \leq\left\|\tilde{T}_{\lambda}\right\|_{S_{1}}\left\|\mathscr{A}_{\lambda}^{-1}\right\|_{S_{1}}\left\|D_{\lambda}^{\prime}\right\|_{S_{1}}\|y\|_{l_{\infty}} .
$$

Then by Lemma 5.3, we successively get

$$
\left\|D_{\lambda}^{\prime}\right\|_{S_{1}}=\sup _{n \geq 1}\left|\frac{1}{\left(a_{n}-\lambda\right)}\right| \leq \begin{cases}\frac{1}{|\lambda|} \sin \theta & \text { for } \lambda=|\lambda| e^{i \theta} \notin \mathbb{R}^{-} \\ \frac{1}{|\lambda|} & \text { for } \lambda \in \mathbb{R}^{-} .\end{cases}
$$

Now as we have seen in (5.9), we have $\left\|I-\mathscr{A}_{\lambda}\right\|_{S_{1}}=\sup _{n \geq n_{0}}\left(\left|\chi_{n}\right|\right) \leq 1 / 2$ and then in the Banach algebra $S_{1}$, we easily get

$$
\left\|\mathscr{A}_{\lambda}^{-1}\right\|_{S_{1}} \leq \sum_{m=0}^{\infty}\left\|\left(I-\mathscr{A}_{\lambda}\right)\right\|_{S_{1}}^{m} \leq \sum_{m=0}^{\infty} 2^{-m}=2 .
$$

Finally, by (5.9), (5.10), and (5.11), we have $\sup _{\lambda \notin \prod_{\varepsilon_{A}}}\left\|\widetilde{T}_{\lambda}\right\|_{S_{1}}<\infty$ and we conclude that $\left\|(A-\lambda I)^{-1}\right\|_{\mathscr{B}\left(c_{0}\right)}^{*} \leq M /|\lambda|$ for all $\lambda \notin \prod_{\varepsilon_{A}}$. We get similar results for $B+\mu I$. This concludes the proof of (iv).

5.2. The case when $A$ and $B$ are matrix maps from $D(A)$ and $D(B)$ into $c$. In this section, $A$ and $B$ satisfy conditions given in (3.5) and (3.6). We will state results similar to those given in Section 5.1, when $c_{0}$ is replaced by $c$. 
12 Matrix and generators of analytic semigroups

Proposition 5.7. (i) Let $\left.\varepsilon_{A} \in\right] 0, \pi / 2[$. For every $\lambda$ with $|\operatorname{Arg}(\lambda)| \geq \varepsilon$, the infinite matrix $A-\lambda I$ considered as an operator in $s_{1 / a}^{(c)}$ is invertible and

$$
(A-\lambda I)^{-1} \in\left(c, s_{1 / a}^{(c)}\right)
$$

(ii) Let $\left.\varepsilon_{B} \in\right] 0, \pi / 2\left[\right.$. For every $\mu$ with $|\operatorname{Arg}(\mu)| \leq \pi-\varepsilon_{B}$, the infinite matrix $B+\mu I$ considered as an operator in $s_{1 / \beta}^{(c)}$ is invertible and

$$
(B+\mu I)^{-1} \in\left(c, s_{1 / \beta}^{(c)}\right)
$$

Proof. (i) First, it can easily be seen that since

$$
1+\frac{b_{n}}{a_{n}-\lambda} \frac{a_{n}}{a_{n+1}} \longrightarrow 1 \quad(n \longrightarrow \infty) \forall \lambda \notin \prod_{\varepsilon_{A}}
$$

and $\mathscr{A}_{\lambda} \in S_{1 / a}$, we have $\mathscr{A}_{\lambda} \in\left(s_{1 / a}^{(c)}, s_{1 / a}^{(c)}\right)$. Then using the notation of Proposition 5.4, there is $n_{0}^{\prime}$ such that

$$
\left\|I-\mathscr{A}_{\lambda}\right\|_{\Re\left(s_{1 / a}^{(c)}\right)}^{*}=\left\|I-\mathscr{A}_{\lambda}\right\|_{S_{1 / a}}^{*}=\sup _{n \geq n_{0}^{\prime}}\left(\left|\chi_{n}\right| \frac{a_{n}}{a_{n+1}}\right) \leq \frac{1}{2} \quad \forall \lambda \notin \prod_{\varepsilon_{A}} .
$$

Then $\mathscr{A}_{\lambda}^{-1} \in \mathscr{B}_{B}\left(s_{1 / a}^{(c)}\right) \cap S_{1 / a}$, and since $\mathscr{A}_{\lambda}^{-1} \in S_{1 / a}$ is an infinite matrix, we have $\mathscr{A}_{\lambda}^{-1} \in$ $\left(s_{1 / a}^{(c)}, s_{1 / a}^{(c)}\right)$. Then for any $y \in c$, we successively get $D_{\lambda}^{\prime} y=\left(y_{n} /\left(a_{n}-\lambda\right)\right)_{n \geq 1} \in s_{1 / a}^{(c)}$, $\mathscr{A}_{\lambda}^{-1}\left(D_{\lambda}^{\prime} y\right) \in s_{1 / a}^{(c)}$, and $\tilde{T}_{\lambda}\left[\mathscr{A}_{\lambda}^{-1}\left(D_{\lambda}^{\prime} y\right)\right] \in s_{1 / a}^{(c)}$. We conclude that $(A-\lambda I)^{-1} \in\left(c, s_{1 / a}^{(c)}\right)$ for every $\lambda$ with $|\operatorname{Arg}(\lambda)| \geq \varepsilon$.

(ii) Reasoning as in Proposition 5.4, there are $M, M^{\prime}>0$ such that for every $\mu \in \Sigma_{B}$,

$$
\begin{gathered}
\left|\frac{\gamma_{2 k}}{\beta_{2 k}+\mu}\right| \frac{\beta_{2 k}}{\beta_{2 k-1}} \leq \frac{M}{\beta_{2 k} \sin \varepsilon_{B}} \frac{\beta_{2 k}}{\beta_{2 k-1}}=o(1) \quad(k \rightarrow \infty), \\
\left|\frac{\gamma_{2 k+1}}{\beta_{2 k+1}+\mu}\right| \frac{\beta_{2 k+1}}{\beta_{2 k}} \leq \frac{M^{\prime}}{\beta_{2 k+1} \sin \varepsilon_{B}} \frac{\beta_{2 k+1}}{\beta_{2 k}}=o(1) \quad(k \longrightarrow \infty) .
\end{gathered}
$$

We deduce that there is $n_{1}^{\prime}$ with

$$
\tau_{1}=\sup _{k \geq n_{1}^{\prime} / 2}\left|\frac{\gamma_{2 k}}{\beta_{2 k}+\mu}\right| \frac{\beta_{2 k}}{\beta_{2 k-1}} \leq \frac{1}{2}, \quad \tau_{2}=\sup _{k \geq\left(n_{1}^{\prime}-1\right) / 2}\left|\frac{\gamma_{2 k+1}}{\beta_{2 k+1}+\mu}\right| \frac{\beta_{2 k+1}}{\beta_{2 k}} \leq \frac{1}{2} .
$$


Now by (5.33), we have

$$
\left|\frac{\gamma_{n}}{\beta_{n}+\mu}\right| \frac{\beta_{n}}{\beta_{n-1}} \longrightarrow 0 \quad(n \rightarrow \infty)
$$

and $\mathscr{B}_{\mu} \in\left(s_{1 / \beta}^{(c)}, s_{1 / \beta}^{(c)}\right)$ for all $\mu \in \Sigma_{B}$. Thus

$$
\begin{aligned}
\left\|I-\mathscr{B}_{\mu}\right\|_{\left(s_{1 / \beta}^{(c)}, s_{1 / \beta}^{(c)}\right)}^{*} & =\left\|I-\mathscr{B}_{\mu}\right\|_{S_{1 / \beta}}^{*}=\sup _{n \geq n_{1}^{\prime}}\left(\left|\frac{\gamma_{n}}{\beta_{n}+\mu}\right| \frac{\beta_{n}}{\beta_{n-1}}\right) \\
& =\max \left(\tau_{1}, \tau_{2}\right) \leq \frac{1}{2}
\end{aligned}
$$

and $\mathscr{B}_{\mu}^{-1} \in\left(s_{1 / \beta}^{(c)}, s_{1 / \beta}^{(c)}\right)$ for all $\mu \in \Sigma_{B}$. Reasoning as in (i) with $(B+\mu I)^{-1}=\mathscr{B}_{\mu}^{-1} \widetilde{R}_{\mu} D_{\mu}^{\prime}$, we conclude that $B+\mu I$ considered as operator from $s_{1 / \beta}^{(c)}$ to $c$ is invertible and $(B+\mu I)^{-1} \in$ $\left(c, s_{1 / \beta}^{(c)}\right)$ for all $\mu \in \Sigma_{B}$. This completes the proof.

We can state the following.

Theorem 5.8. In the space $c$, the two linear operators $A$ and $B$ are closed and satisfy the following:

(i) $D(A)=c(A)=s_{1 / a}^{(c)}$,

(ii) $D(B)=c(B)=s_{1 / \beta}^{(c)}$,

(iii) $\overline{D(A)} \neq c, \overline{D(B)} \neq c$,

(iv) there are $\varepsilon_{A}, \varepsilon_{B}>0$ (with $\varepsilon_{A}+\varepsilon_{B}<\pi$ ) such that

$$
\begin{gathered}
\left\|(A-\lambda I)^{-1}\right\|_{\mathscr{B}(c)}^{*} \leq \frac{M}{|\lambda|} \quad \forall \lambda \neq 0, \quad|\operatorname{Arg}(\lambda)| \geq \varepsilon_{A}, \\
\left\|(B+\mu I)^{-1}\right\|_{\mathscr{B}(c)}^{*} \leq \frac{M}{|\mu|} \quad \forall \mu \neq 0, \quad|\operatorname{Arg}(\mu)| \leq \pi-\varepsilon_{B} .
\end{gathered}
$$

Proof. Show that $A$ is a closed operator. For this, consider a sequence $x_{p}^{\prime}=\left(x_{n p}\right)_{n \geq 1}$ tending to $x=\left(x_{n}\right)_{n \geq 1}$ in $c$, as $p$ tends to infinity, where $x_{p}^{\prime} \in s_{1 / a}^{(c)}$ for all $p$. Then $A x_{p}^{\prime} \rightarrow y(p \rightarrow \infty)$ in $c$, that is, $A_{n}\left(x_{p}^{\prime}\right) \rightarrow A_{n}(x)=y_{n}(p \rightarrow \infty)$ for all $n$. It remains to show that $x \in s_{1 / a}^{(c)}$. For this, note that since $b \in c$ and $x \in c$, we conclude that $a_{n} x_{n}=y_{n}-b_{n} x_{n+1}$ tends to a limit as $n$ tends to infinity. The proof for $B$ is similar. The proof of statements (i) and (ii) comes from Proposition 4.3 and follows the same lines as that for Theorem 5.6(i), (ii).

(iii) Follows exactly the same lines as that in the proof given in the case when $E=l_{\infty}$ in [15, Proposition 3, page 196].

The proof of (iv) is a consequence of Proposition 5.7(i) and follows exactly the same lines as that in the proof of Theorem 5.6.

\section{Acknowledgments}

The authors thank the referee for his contribution to improve the presentation of the paper. The work of the second author is supported by the Agence Universitaire de la Francophonie (AUF). 


\section{References}

[1] B. Altay and F. Başar, On the fine spectrum of the generalized difference operator $B(r, s)$ over the sequence spaces $c_{0}$ and $c$, International Journal of Mathematics and Mathematical Sciences 2005 (2005), no. 18, 3005-3013.

[2] G. Da Prato and P. Grisvard, Sommes d'opérateurs linéaires et équations différentielles opérationnelles, Journal de Mathématiques Pures et Appliquées. Neuvième Série 54 (1975), no. 3, 305387.

[3] B. de Malafosse, Some properties of the Cesàro operator in the space $s_{r}$, Faculty of Sciences. University of Ankara. Series A1. Mathematics and Statistics 48 (1999), no. 1-2, 53-71 (2000).

[4] __ Application of the sum of operators in the commutative case to the infinite matrix theory, Soochow Journal of Mathematics 27 (2001), no. 4, 405-421.

[5] __ Properties of some sets of sequences and application to the spaces of bounded difference sequences of order $\mu$, Hokkaido Mathematical Journal 31 (2002), no. 2, 283-299.

[6] _ On matrix transformations and sequence spaces, Rendiconti del Circolo Matematico di Palermo. Serie II 52 (2003), no. 2, 189-210.

[7] _ On some BK spaces, International Journal of Mathematics and Mathematical Sciences 2003 (2003), no. 28, 1783-1801.

[8] On the sets of sequences that are strongly $\alpha$-bounded and $\alpha$-convergent to naught with index $p$, Seminario Matematico. Universitàe Politecnico di Torino 61 (2003), no. 1, 13-32.

[9] 57 (2005), no. 1-2, 41-60.

[10] B. de Malafosse and V. Rakocevic, Applications of measure of noncompactness in operators on the spaces $s_{\alpha}, s_{\alpha}^{0}, s_{\alpha}^{(c)}$ and $l_{\alpha}^{p}$, to appear in Journal of Mathematical Analysis and Applications.

[11] M. Fuhrman, Sums of operators of parabolic type in a Hilbert space: strict solutions and maximal regularity, Advances in Mathematical Sciences and Applications 4 (1994), no. 1, 1-34.

[12] P. Grisvard, Commutativité de deux foncteurs d'interpolation et applications, Journal de Mathématiques Pures et Appliquées. Neuvième Série 45 (1966), 143-206.

[13] A. M. Jarrah and E. Malkowsky, Ordinary, absolute and strong summability and matrix transformations, Filomat 2003 (2003), no. 17, 59-78.

[14] T. Kato, Perturbation Theory for Linear Operators, Die Grundlehren der mathematischen Wissenschaften, vol. 132, Springer, New York, 1966.

[15] R. Labbas and B. de Malafosse, An application of the sum of linear operators in infinite matrix theory, Faculty of Sciences. University of Ankara. Series A1. Mathematics and Statistics 46 (1997), no. 1-2, 191-210 (1998).

[16] R. Labbas and B. Terreni, Somme d'opérateurs linéaires de type parabolique. I, Bollettino. Unione Matematica Italiana. B. Serie VII 1 (1987), no. 2, 545-569.

[17] _ Sommes d'opérateurs de type elliptique et parabolique. II. Applications, Bollettino. Unione Matematica Italiana. B. Serie VII 2 (1988), no. 1, 141-162.

[18] A. Pazy, Semigroups of Linear Operators and Applications to Partial Differential Equations, Applied Mathematical Sciences, vol. 44, Springer, New York, 1983.

[19] A. Wilansky, Summability Through Functional Analysis, North-Holland Mathematics Studies, vol. 85, North-Holland, Amsterdam, 1984.

Bruno de Malafosse: Laboratoire de Mathématiques Appliquées du Havre (LMAH), Université du Havre, BP 4006 IUT Le Havre, 76610 Le Havre, France

E-mail address: bdemalaf@wanadoo.fr

Ahmed Medeghri: Laboratoire de Mathématiques Pures et Appliquées, Université de Mostaganem, BP 227, Mostaganem 27000, Algeria

E-mail address: medeghri@univ-mosta.dz 